P. C. $\mathrm{Hu}$

Nagoya Math. J.

Vol. 120 (1990), 171-180

\title{
HOLOMORPHIC MAPPING INTO ALGEBRAIC VARIETIES OF GENERAL TYPE, II
}

\section{PEICHU HU}

This announcement is a continuation of $\mathrm{Hu}$ [3]. Our results improve Theorem 1 of [3], but the latter is needed in the proof of the former.

Let $f: M \rightarrow N$ be a holomorphic mapping from a connected complex manifold $M$ of dimension $m$ to a projective algebraic manifold $N$ of dimension $n$. Assume that $M$ possess a parabolic exhaustion $\tau$ and denote

$$
\begin{aligned}
& v=d d^{c} \tau, \sigma=d^{c} \log \tau \wedge\left(d d^{c} \log \tau\right)^{m-1}, \\
& A(t ; \zeta)=t^{2-2 m} \int_{M[t]} \zeta \wedge v^{m-1}, T(r, s ; \zeta)=\int_{s}^{r} \frac{A(t ; \zeta)}{t} d t,
\end{aligned}
$$

where $\zeta$ is a form of bidegree $(1,1)$ on $M$ and $M[t]=\left\{x \in M: \tau(x) \leq t^{2}\right\}$. Suppose throughout that $L$ is a positive holomorphic line bundle over $N$ with a hermitian metric $\rho$ along the fibers of $L$ such that the Chern form $c(L, \rho)>0$. The characteristic function of $f$ for $(L, \rho)$ is defined by

$$
T(r, s)=T\left(r, s ; f^{*}(c(L, \rho))\right) .
$$

Let $\operatorname{Ric}_{\tau}(r, s)$ be the Ricci function of $\tau$. We obtain that

Theorem 1. Let $N$ be of general type. If $M$ is a Stein, covering parabolic space of $C^{m}$ and if rank $f=\min (m, n)$, then there exist positive constants $c_{1}$ and $c_{2}$ such that

$$
c_{1} T(r, s) \leq \operatorname{Ric}_{\tau}(r, s)+c_{2} \log r
$$

with the exception of a set of values $(r)$ of finite measure.

CoROLLARY 2. If $N$ is of general type, any non-degenerate holomorphic mappings $f: C^{m} \rightarrow N$ is necessarily rational.

In fact, we will prove a more general result than Theorem 1 (see Theorem 4). For this, we need some facts about hermitian geometry, dual classification map, associated maps and covering space.

Received November 14, 1989. 
a) Hermitian Geometry

Let $V$ be a complex vector space of dimension $n+1$. Then $V^{*}$ is the dual vector space, $\wedge V$ is the exterior product. The Grassmann cone in $\wedge V$ is defined by ${ }^{k} \tilde{G}_{k}(V)=\left\{a_{0} \wedge \cdots \wedge a_{k}: a_{i} \in V\right\}$ with $\tilde{G}_{0}(V)=V$ and $\tilde{G}_{n}(V) \approx C$. If $0 \neq x \in V$, let $\boldsymbol{P}(x)=C x$ be the complex line spanned by $x$. If $A \subseteq V$, define $\boldsymbol{P}(A)=\{\boldsymbol{P}(x): 0 \neq x \in A\}$. Then $\boldsymbol{P}(V)$ is the complex projective space associated to $V$. A holomorphic map $\boldsymbol{P}: V-\{0\} \rightarrow \boldsymbol{P}(V)$ is defined. The same symbol $\boldsymbol{P}$ is used for all vector spaces. Take an integer $k$ with $0 \leq k \leq n$. The Grassmann manifold $G_{k}(V)=\boldsymbol{P}\left(\tilde{G}_{k}(V)\right)$ of order $k$ is a connected, smooth, compact submanifold of $\boldsymbol{P}(\bigwedge V)$. Take $a \in G_{k}(V)$. Then $\tilde{a}=a_{0} \wedge \cdots \wedge a_{k} \neq 0$ exists such that $P(\tilde{a})=a$. A $(k+1)$-dimensional linear subspace $E(a)=C a_{0}+\cdots+C a_{k}$ is associated to $a$, independent of the choice of $a$. The associated projective space $\ddot{E}(a)=\boldsymbol{P}(E(a))$ is smoothly imbedded into $\boldsymbol{P}(V)$ and called a $k$-plane.

Take $a \in G_{k}\left(V^{*}\right)$. Then $\alpha=\alpha_{0} \wedge \cdots \wedge \alpha_{k} \neq 0$ exists such that $\boldsymbol{P}(\alpha)$ $=a . \mathrm{A}(k+1)$-codimensional linear subspace

$$
E[a]=\bigcap_{j=0}^{k} \alpha_{j}^{-1}(0)
$$

and $a(n-k-1)$-plane $\ddot{E}[a]=\boldsymbol{P}(E[a])$ are associated to $a$. The biholomorphic dualism map $\delta: G_{k}(V) \rightarrow G_{n-k-1}\left(V^{*}\right)$ is defined by $E[\delta(a)]=E(a)$.

The trivial bundle $G_{k}(V) \times V$ contains the tautological bundle

$$
S_{k}(V)=\left\{(a, x) \in G_{k}(V) \times V: x \in E(a)\right\}
$$

as a holomorphic subbundle. The quotient bundle $Q_{k}(V)$ exists and the classifying sequence

$$
0 \rightarrow S_{k}(V) \rightarrow G_{k}(V) \times V \rightarrow Q_{k}(V) \rightarrow 0
$$

is obtained. If $q=n-k-1$, then (1) is the pullback of

$$
0 \rightarrow Q_{q}\left(V^{*}\right)^{*} \rightarrow G_{q}\left(V^{*}\right) \times V \rightarrow S_{q}\left(V^{*}\right)^{*} \rightarrow 0
$$

under the dualism $\delta: G_{k}(V) \rightarrow G_{q}\left(V^{*}\right)$.

Let $l$ be a hermitian metric on $V$. Then $l$ induces hermitian metrics $l$ along the fibers of $Q_{q}\left(V^{*}\right)^{*}, G_{q}\left(V^{*}\right) \times V$ and $S_{q}\left(V^{*}\right)^{*}$ and Fubini-Kaehler forms $\Omega_{q}>0$ on $G_{q}\left(V^{*}\right)$. Then

$$
\begin{aligned}
& c\left(S_{0}\left(V^{*}\right)^{*}, l\right)=\Omega_{0}, \\
& \operatorname{Ric}\left(\Omega_{0}^{n}\right)=-(n+1) \Omega_{0},
\end{aligned}
$$


b) Dual classification map

A holomorphic vector bundle homomorphism $\xi: N \times V \rightarrow E$ is said to be ample at $x \in N$, if $\xi(\{x\} \times V)=E_{x}$, where $E$ is a holomorphic vector bundle over $N$. The set $N_{\infty}$ of all $x \in N$ such that $\xi$ is ample at $x$ is open. Also $N-N_{\infty}$ is analytic. Then $\xi$ is said to be an amplification if $N=N_{\infty}$, semi-amplification if $N-N_{\infty}$ is thin (see Stoll [5]).

Abbreviate the tensor product $L^{\otimes p}$ by $L^{p}$. We say that $L$ is ample if there exists some $p$ such that a basis of sections $\left(s_{0}, \cdots, s_{k}\right)$ of $H^{0}\left(N, L^{p}\right)$ generates $L^{p}$ at every point (i.e., the evaluation map e: $N \times H^{0}\left(N, L^{p}\right) \rightarrow L^{p}$ defined by $e(x, s)=s(x)$ is an amplification), and give a projective imbedding

$$
\left(s_{0}, \cdots, s_{k}\right): N \rightarrow P\left(H^{0}\left(N, L^{p}\right)\right) .
$$

We say that $L$ is very ample if we can take $p=1$ in the above condition (see S. Lang [4]). Let $L$ be ample. We have a projective imbedding (3). Hence we can take a complex vector subspace $V$ of $H^{0}\left(N, L^{p}\right)$ with $\operatorname{dim} V$ $=n+1$ such that the evaluation map $e: N \times V \rightarrow L^{p}$ is an amplification (see Stoll [5], Lemma 16.1, Proposition A16). Let $S$ be the kernel of $e$. An exact sequence

$$
0 \rightarrow S \rightarrow N \times V \rightarrow L^{p} \rightarrow 0
$$

is defined. Here $S$ has fiber dimension $n$. If $x \in N$, one and only one $\varphi(x) \in \boldsymbol{P}\left(V^{*}\right)$ and $\varphi_{0}(x) \in G_{n-1}(V)$ exist such that

$$
E[\varphi(x)]=S_{x}=E\left(\varphi_{0}(x)\right) .
$$

The maps $\varphi_{0}: N \rightarrow G_{n-1}(V)$ and $\varphi ; N \rightarrow \boldsymbol{P}\left(V^{*}\right)$ are called the classification map and the dual classification map respectively, which are holomorphic. If $\delta$ is the dualism, then $\varphi=\delta \circ \varphi_{0}$. The classification map $\varphi_{0}$ pulls back (1) to (4) for $k=n-1$. Hence

$$
L^{p}=\left(\varphi_{0}\right)^{*}\left(Q_{n-1}(V)\right)=\varphi_{*}^{*}\left(S_{0}\left(V^{*}\right)^{*}\right) .
$$

Let $l$ be a hermitian metric on $V$. Then $l$ induces hermitian metrics $l$ along the fibers of $S, N \times V$ and $L$ by (4) and along $Q_{0}\left(V^{*}\right)^{*}, P\left(V^{*}\right) \times V$ and $S_{0}\left(V^{*}\right)^{*}$ by (2) for $q=0$. Hence

$$
\begin{aligned}
& \operatorname{pc}(L, l)=c\left(L^{p}, l^{p}\right)=\varphi^{*}\left(c\left(S_{0}\left(V^{*}\right)^{*}, l\right)\right)=\varphi^{*}\left(\Omega_{0}\right), \\
& \operatorname{Ric} c\left(L^{p}, l^{p}\right)^{n}=\operatorname{Ric} \varphi^{*}\left(\Omega_{0}^{n}\right)=\varphi^{*}\left(\operatorname{Ric} \Omega_{0}^{n}\right)=-(n+1) c\left(L^{p}, l^{p}\right) .
\end{aligned}
$$

c) Associated maps 
Now we consider the holomorphic map $\varphi_{f}=\varphi \circ f: M \rightarrow \boldsymbol{P}\left(V^{*}\right)$, where $\varphi: N \rightarrow \boldsymbol{P}\left(V^{*}\right)$ is the dual classification map in b). Let $L_{f}$ is the pullback $\varphi_{f}^{*}\left(S_{0}\left(V^{*}\right)^{*}\right)$ of the hyperplane section bundle $S^{0}\left(V^{*}\right)^{*}$ on $\boldsymbol{P}\left(V^{*}\right)$. Take a holomorphic form $B$ of bidegree $(m-1,0)$ on $M$. We can define the $k^{\text {th }}$ representation section $F_{k}$ of $\varphi_{f}$ of the holomorphic vector bundle

$$
L_{f}[k]=\left(M \times\left(\bigwedge_{k+1} V^{*}\right)\right) \otimes\left(L_{f}\right)^{k+1} \otimes\left(K_{M}\right)^{k(k+1) / 2}
$$

by means of the $B$-derivative, where $K_{M}$ is the canonical bundle of $M$. Here

$$
F_{0}: M \rightarrow L_{f}[0]=\left(M \times V^{*}\right) \otimes L_{f}
$$

but $F_{k} \equiv 0$ if $k>n$. If $F_{k} \equiv 0$, then $F_{k+1} \equiv 0$. Hence an integer $l_{f}$ exists uniquely such that $F_{k} \not \equiv 0$ if $0 \leq k \leq l_{f}$ and $F_{k} \equiv 0$ if $k>l_{f}$. We call $l_{f}$ the generality index of $\varphi_{f}$ for $B$. The map $\varphi_{f}$ is said to be general for $B$ if $l_{f}=n$ (see Stoll [6]). If $M$ admits $m$ analytically independent holomorphic functions, then for any finite sets of meromorphic maps defined on $M$, there exists a holomorphic form $B$ of degree $m-1$ on $M$ such that the generality index of each of these maps $\varphi_{f}$ for $B$ equals the dimension of the smallest projective plane containing the image of $\varphi_{s}$ (see Stoll [7], Theorem 7.11).

For each $k$ with $0 \leq k \leq l_{f}$, the $k^{\text {th }}$ associated map

$$
f_{k}=\boldsymbol{P} \circ F_{k}: M \rightarrow G_{k}\left(V^{*}\right)
$$

of $\varphi_{f}$ is defined with $f_{0}=\varphi_{f}$, and is holomorphhic. Define

$$
L_{f}[-1]=M \times C
$$

and let $F_{-1}$ be the trivial section defined by $F_{-1}(z)=(z, 1)$. Denote the divisor of $F_{k}$ by $\mu_{F_{k}}$. Then $\mu_{F_{-1}}=\mu_{F_{0}}=0$. For $0 \leq k \leq l_{f}$, teh $k^{\text {th }}$ stationary divisor

$$
D_{f_{k}}=\mu_{F_{k-1}}-2 \mu_{F_{k}}+\mu_{k+1} \geq 0
$$

is non-negative (effective).

Define

$$
H_{k}=m i_{m-1} f_{k}^{*}\left(\Omega_{k}\right) \wedge B \wedge \bar{B} \geq 0
$$

with $H_{k}=0$ if $k<0$ or if $k \geq l_{f}$. For $0 \leq k<l_{f}$, we have the identity

$$
\operatorname{Ric} H_{k}=f_{k-1}^{*}\left(\Omega_{k-1}\right)-2 f_{k}^{*}\left(\Omega_{k}\right)+f_{k+1}^{*}\left(\Omega_{k+1}\right) .
$$


Since $M$ is a parabolic manifold, the open set

$$
M^{+}=\{x \in M: v(x)>0\}
$$

is not empty. On $M^{+}$, an on-negative function $h_{k}$ is defined by $H_{k}=h_{k}^{2} v^{m}$. Abbreviate

$$
T_{k}(r, s)=T\left(r, s ; f_{k}^{*}\left(\Omega_{k}\right)\right) .
$$

Then for almost all $s, r$ with $0<s<r$, we have the Plücker Difference Formula

$$
\begin{aligned}
& N\left(r, s ; D_{f_{k}}\right)+T_{k-1}(r, s)-2 T_{k}(r, s)+T_{k+1}(r, s) \\
& \quad=B\left(r, s ; h_{k}^{2}\right)+\operatorname{Ric}_{\tau}(r, s),
\end{aligned}
$$

where $T_{k}(r, s)=0$ if $k<0$ or $k \geq l_{f}$, and

$$
\begin{aligned}
& B(t, h)=\frac{1}{2} \int_{\partial M[t]}(\log h)_{\sigma}, B(r, s ; h)=B(r, h)-B(s, h), \\
& N(r, s ; D)=\int_{s}^{r} n(t, D) \frac{d t}{t}, n(t, D)=t^{2-2 m} \int_{D \cap M[t]} v^{m-1} .
\end{aligned}
$$

The exhaustion $\tau$ is said to majorize the holomorphic form $B$ of degree $m-1$, if for every $r>0$ there exists a constant $c \geq 1$ such that

$$
0 \leq m i_{m-1} B \wedge \bar{B} \leq c v^{m-1} \quad \text { on } M[r]
$$

where

$$
i_{m-1}=(-1)^{(m-1)(m-2) / 2}\left(\frac{\sqrt{-1}}{2 \pi}\right)^{m-1}(m-1) !
$$

The infimum of all these constants is called $Y_{0}(r)$. Then $Y_{0}(r) \geq 1$, and increases. Define

$$
Y(r)=\lim _{r<t \rightarrow r} Y_{0}(t) .
$$

Then $Y(r) \geq \mathrm{Y}_{0}(r) \geq 1$. The increasing function $Y$ is called the majorant associated to $\tau$ and $B$. If $r>0$, then

$$
\begin{array}{ll}
m i_{m-1} B \wedge \bar{B} \leq Y(r) v^{m-1} & \text { on } M[r] \\
m i_{m-1} B \wedge \bar{B} \leq(Y \circ \sqrt{\tau}) v^{m-1} & \text { on } M .
\end{array}
$$

If $m=1$, that is, if $M$ is an open parabolic Riemann surface, we take $B=1$, then $m i_{m-1} B \wedge \bar{B}=v^{m-1}$ and $\tau$ majorizes $B$ with $Y \equiv 1$. From now, we assume that $\tau$ majorizes $B$ with $Y$. We use the notation 


$$
\|_{\varepsilon} a(r) \leq b(r)
$$

to mean that the stated inequality holds except on an open set $I \subset R^{+}$ such that $\int_{I} r^{\varepsilon} d r<\infty$ for $\varepsilon>0$. We have

$$
\begin{aligned}
& \|_{\varepsilon} B\left(r, h_{k}^{2}\right) \leq \frac{\mathfrak{c}}{2}(1+\varepsilon)^{2}\left(\log T_{k}(r, s)+\log Y(r)\right)+\frac{\mathfrak{c}}{2} \varepsilon \log r, \\
& \|_{\varepsilon} T_{k}(r, s) \leq 3^{k} T_{0}(r, s)+\frac{3^{k}-1}{2}\left(\log Y(r)+\operatorname{Ric}_{\tau}(r, s)+\varepsilon \mathfrak{c} \log r\right),
\end{aligned}
$$

where the constant $c$ is the volume of $\partial M[r]$ (see Stoll [6], Proposition 6.14 6.15). (9) and (10) imply

$$
\begin{aligned}
& \|_{\varepsilon} B\left(r, s ; h_{k}^{2}\right) \leq \frac{\mathfrak{c}}{2}(1+\varepsilon)^{3}\left(\log T_{0}(r, s)+\log Y(r)\right. \\
& \left.\quad+\log ^{+} \operatorname{Ric}_{\mathfrak{\tau}}(r, s)\right)+\varepsilon \mathfrak{c} \log r .
\end{aligned}
$$

d) Covering space

If $(M, \tau)$ is a covering parabolic space of $\left(C^{m}, \tau_{0}\right)$ where $\tau_{0}(z)=|z|^{2}$, then there is a proper surjective holomorphic map

$$
\beta=\left(\beta_{1}, \cdots, \beta_{m}\right): M \rightarrow C^{m}
$$

such that $\tau=\tau_{0} \circ \beta=|\beta|^{2}$. The divisor of $d \beta_{1} \wedge \cdots \wedge d \beta_{m} \not \equiv 0$ is called the branching divisor of $\beta$ and denoted by $D_{\beta}$. Then

$$
\operatorname{Ric}_{\imath}(r, s)=N\left(r, s ; D_{\beta}\right) \geq 0 .
$$

Define $S=\operatorname{supp} D_{\beta}$. Then $\beta(S)$ is an analytic subset of $C^{m}$. Let $S_{0}$ be the $(m-1)$-dimensional component of $\beta(S)$. If $S_{0}$ is affine algebraic of degree $d$, then we have

$$
\operatorname{Ric}_{r}(r, s)=N\left(r, s ; D_{\beta}\right) \leq d c \log \frac{r}{s}
$$

for $0<s<r$. If $\beta$ is biholomorphic, $\operatorname{Ric}_{\tau}(r, s) \equiv 0$.

If $\varphi_{f}$ is linearly non-degenerate, then there is a holomorphic form $\hat{B}$ of bidegree $(m-1,0)$ on $C^{m}$ whose coefficients are polynomials of at most degree $n-1$, such that $\varphi_{f}$ is general for $B=\beta^{*}(\hat{B})$. Hence there is a constant $c>0$ such that $\tau$ majorizes $B$ with

$$
Y(r) \leq 1+c r^{2 n-2} \quad \text { for } r \geq 1 \text {. }
$$

see Stoll [6]. 
If $M=\boldsymbol{C}^{m}$ and $\varphi_{f}\left(\boldsymbol{C}^{m}\right)$ does not be contained in any hyperplanes of $P\left(V^{*}\right)$, there is a holomorphic form $B$ of degree $m-1$ on $C^{m}$ whose coefficients are constants such that $\varphi_{f}$ is general for $B$ and such that $\tau_{0}$ majorizes $B$ with

$$
Y(r) \leq c
$$

for a constant $c$.

e) Main results

Let $\psi$ be a positive form of class $C^{\infty}$ and bidegree $(1,1)$ on $N$ such that

$$
\varlimsup_{r \rightarrow \infty} \log T\left(r, s ; f^{*}(\psi)\right) / T(r, s)=0
$$

Define

$$
\ddot{\psi}_{f}=m i_{m-1} f^{*}(\psi) \wedge B \wedge \bar{B}, e_{f}=f^{*}\left(\operatorname{Ric} \psi^{n}\right)-n \operatorname{Ric} \ddot{\psi}_{f}
$$

and define $\eta$ by $\ddot{\psi}_{f}=\eta f^{*}(\psi) \wedge v^{m-1}$. Let

$$
E_{f}(r, s)=T\left(r, s ; e_{f}\right)+n B(r, s ; \eta) .
$$

In [3], we proved that

THEOREM A. Let $N$ be of general type. If there exists an effective Jacobian section of $f$ and if $\operatorname{rank} f=\min (m, n)$, then exist positive constants $c_{1}$ and $c_{2}$ such that

$$
\|_{\varepsilon} c_{1} T(r, s) \leq n \operatorname{Ric}_{\varepsilon}(r, s)+E_{f}(r, s)-n N\left(r, s ; D_{f}\right)+c_{2} \varepsilon \log r,
$$

where $D_{f}$ is the divisor of $\ddot{\psi}_{f}$.

Abbreviate

$$
n_{k}(t)=n\left(t, \mu_{F_{k}}\right), N_{k}(r, s)=N\left(r, s ; \mu_{F_{k}}\right)
$$

for the $k^{\text {th }}$ representation section $F_{k}$ of $\varphi_{f}$. We have

TheORem 3. Let $L$ be an ample, positive holomorphic line bundle over $N$ with the projective imbedding (3). Assume that $B$ is a holomorphic form of bidegree $(m-1,0)$ on $M$ such that $\tau$ majorizes $B$ with $Y$ and such that $\varphi_{f}$ is general for $B$. Then for $\psi=p c(L, l)$, we have

$$
\begin{aligned}
& \|_{\varepsilon} E_{f}(r, s)-n N\left(r, s ; D_{f}\right) \leq-N_{n}(r, s) \\
& \quad+\frac{n c}{2} \log Y(r)+\frac{n(n-1)}{2} Q_{\varepsilon}(r)-n B(s, \eta),
\end{aligned}
$$


where

$$
\begin{aligned}
& Q_{\varepsilon}(r)=\frac{\mathfrak{c}}{2}(1+\varepsilon)^{3}\left(\log T(r, s)+\log Y(r)+\log ^{+} \operatorname{Ric}_{\tau}(r, s)\right) \\
& +\operatorname{Ric}_{\tau}(r, s)+2 \mathrm{c} \varepsilon \log r .
\end{aligned}
$$

Proof. Note that

$$
\begin{aligned}
T_{0}(r, s) & =T\left(r, s ; f^{*}(\psi)\right)=p T\left(r, s ; f^{*}(c(L, l))\right) \\
& =p T(r, s)+0(1)
\end{aligned}
$$

See Stoll [5], Theorem 12.5. Hence (6), (8) and (11) imply

$$
\begin{aligned}
\|_{\varepsilon} N_{k-1}(r, s) & +T_{k-1}(r, s)-2\left(N_{k}(r, s)+T_{k}(r, s)\right) \\
& +N_{k+1}(r, s)+T_{k+1}(r, s) \leq Q_{\varepsilon}(r)
\end{aligned}
$$

Multiply (20) by $(n-k)$ and add these for $k=1, \cdots, n-1$. We get

$$
\begin{aligned}
\|_{\varepsilon}(n-1) T_{0}(r, s)-n T_{1}(r, s) & \leq n N_{1}(r, s)-N_{n}(r, s) \\
& +\frac{n(n-1)}{2} Q_{\varepsilon}(r) .
\end{aligned}
$$

Now $\ddot{\psi}_{f}=H_{0},(5)$ and (7) imply

$$
\begin{aligned}
f^{*}\left(\operatorname{Ric} \psi^{n}\right)-n \operatorname{Ric} \ddot{\psi}_{f} & =-(n+1) f_{0}^{*}\left(\Omega_{0}\right)-n \operatorname{Ric} H_{0} \\
& =(n-1) f_{0}^{*}\left(\Omega_{0}\right)-n f_{1}^{*}\left(\Omega_{1}\right),
\end{aligned}
$$

which yields

$$
T\left(r, s ; e_{f}\right)=(n-1) T_{0}(r, s)-n T_{1}(r, s) .
$$

Since $\tau$ majorizes $B$ with $Y$, we obtain

$$
\eta f^{*}(\psi) \wedge v^{m-1}=\ddot{\psi}_{f} \leq(Y \circ \sqrt{\tau}) f^{*}(\psi) \wedge u^{m-1},
$$

which implies $\eta \leq Y \circ \sqrt{\tau}$. Also we have

$$
N\left(r, s ; D_{f}\right)=N\left(r, s ; D_{f_{0}}\right)=N_{1}(r, s)
$$

by (6) and the definition of $D_{f}$ ad $D_{f_{0}}$ for $\psi=c\left(L^{p}, l^{p}\right)$. So (17) follows from (21)-(23). $\quad$ Q.E.D.

Take $\psi=c\left(L^{p}, l^{p}\right)$ in Theorem A. Then (15) follows from (19). Hence Theorem A and 3 imply

TheOREM 4. Let $N$ be of general type. Let $B$ be a holomorphic form of bidegree $(m-1,0)$ on $M$ such that $\tau$ majorizes $B$ with $Y$ and such that 
$\varphi_{f}$ is general for $B$. If there exists an effective Jacobian section of $f$ and if rank $f=\min (m, n)$, then exist positive constants $c_{1}$ and $c_{2}$ such that

$$
\begin{aligned}
& \|_{\varepsilon} N_{n}(r, s)+c_{1} T(r, s) \leq \frac{n(n+1)}{2} \operatorname{Ric}_{\tau}(r, s) \\
& \quad+\frac{n(n+1) c}{4}(1+\varepsilon)^{3}\left(\log Y(r)+\log ^{+} \operatorname{Ric}_{\tau}(r, s)\right)+c_{2} \varepsilon \log r .
\end{aligned}
$$

If $M$ is Stein and $\operatorname{rank} f=\min (m, n)$, effective Jacobian sections exist by Stoll [5], Theorem 14.1, 14.2. Hence (12), (14) and Theorem 4 imply Theorem 1.

Abbreviate

$$
A(t)=A\left(t ; f^{*}(c(L, \rho))\right)
$$

and define

$$
R_{\tau}=\lim _{r \rightarrow \infty} \frac{\operatorname{Ric}_{\tau}(r, s)}{\log r}, \quad Y_{B}=\lim _{r \rightarrow \infty} \frac{\log Y(r)}{\log r}
$$

Hence Theorem 4 with $\varepsilon \rightarrow 0$ implies.

$$
n_{n}(\infty)+c_{1} A(\infty) \leq \frac{n(n+1)}{2} R_{\tau}+\frac{n(n+1) c}{4} Y_{B} .
$$

f) Green-Griffiiths' Conjecture

If $M$ is an irreducible, affine algebraic variety with $A(\infty)<\infty$, then $f$ is rational (Griffiths-King [2], Proposition 5.9, Carlson-Griffiths [1], Proposition 6.20 and Stoll [5], Theorem 20.6). Hence (14) and (25) imply Corollary 2 .

If $M=C^{m}$, then (14)' and (25) yield $A(\infty)=0$, which implies that

Corollary 5. If $N$ is of general type, then the image of any holomorphic map $f: C^{m} \rightarrow N$ with rank $f=\min (m, n)$ is contained in a proper subvariety.

Proof. If not, then $\varphi_{f}$ is linearly non-degenerate. Hence there is a holomorphic form $B$ of degree $m-1$ on $C^{m}$ such that $\varphi_{f}$ is general for $B$ and such that $\tau_{0}$ majorizes $B$ with $(14)^{\prime}$. Since $\operatorname{rank} f=\min (m, n)$ and $c(L, \rho)>0$

$$
A(\infty)=\lim _{r \rightarrow \infty} A(r)>0
$$

which contradicts $A(\infty)=0$.

Q.E.I). 
Corollary 5 implies the following

Green-Griffiths' Conjecture. Let $N$ be of general type (or pseudo canonical). Let $f: C \rightarrow N$ be holomoprhic non-constant. Then the image of $f$ is contained in a proper subvariety.

For more detail, see S. Lang [4].

\section{REFERENCES}

[1] J. Carlson and $\mathrm{Ph}$. Griffiths, A defect relation for equidimensional holomorphic mappings between algebraic varieties, Ann. of Math., (2) 95 (1972), 557-584.

[2] Ph. Griffiths and J. King, Nevanlinna theory and holomorphic mappings between algebraic varieties, Acta. Math., 130 (1973), 145-220.

[ 3 ] P. C. Hu, Holomorphic mapping into algebraic varieties of general type. (to appear)

[4] S. Lang, Hyperbolic and Diophantine analysis. Bull. of Amer, Math., Soc. 14 (1986) , 158-205.

[5] W. Stoll, Value distribution on parabolic spaces. Lecture Notes in Math., 600 (1977), Springer-Verlag.

[6] — Value distribution theory for meromorphic maps. Aspects of Math., E7 (1985), Vieweg.

[7] Deficit and Bezout estimates. Value Distribution Theory. Part B. Pure and Appl, Math., 25 (1973), New York.

Department of Mathematics

Shandong University

Jinan, Shandong,

China 